\title{
An experimental investigation of energy absorption in TRIP steel under impact three-point bending deformation
}

\author{
Hang Pham ${ }^{1,3, a}$ and Takeshi Iwamoto ${ }^{2}$ \\ ${ }^{1}$ Graduate School of Engineering, Hiroshima University, 1-4-1 Kagamiyama, Higashi-Hiroshima, Hiroshima 739-8527, \\ Japan \\ ${ }^{2}$ Institute of Engineering, Hiroshima University, 1-4-1 Kagamiyama, Higashi-Hiroshima, Hiroshima 739-8527, Japan \\ ${ }^{3}$ Faculty of Engineering, Vietnam National University of Agriculture, Trauquy, Gialam, Hanoi 131004, Vietnam
}

\begin{abstract}
TRIP (Transformation-induced Plasticity) steel is nowadays in widespread use in the automobile industry because of their favorable mechanical properties such as high strength, excellent formability and toughness because of strain-induced martensitic transformation. Moreover, when TRIP steel is applied to the components of the vehicles, it is expected that huge amount of kinetic energy will be absorbed into both plastic deformation and martensitic transformation during the collision. Basically, bending deformation due to buckling is one of the major crash deformation modes of automobile structures. Thus, an investigation of energy absorption during bending deformation at high impact velocity for TRIP steel is indispensable. Although TRIP steel have particularly attracted the recent interest of the scientific community, just few studies can be found on the energy absorption characteristic of TRIP steel, especially at impact loading condition. In present study, experimental investigations of bending deformation behaviors of TRIP steel are conducted in the three-point bending tests for both smooth and pre-cracked specimen. Then, energy absorption characteristic during plastic deformation and fracture process at high impact velocity in TRIP steel will be discussed.
\end{abstract}

\section{Introduction}

Over the past century, the most important and challenging task for design considerations in the automotive industry is vehicle crashworthiness and an occupant safety with a decrease in weight of automobiles. Thus, energyabsorption characteristic of structural materials has become a critical issue to protect the passengers from a high-speed crash of automobiles. During a crash of automobiles, a dominant mechanism to keep the safety in automobiles by structures upon the collision is buckling because kinetic energy with higher speed of the vehicles can be consumed by inelastic deformation of components for protection of the passengers [1]. Bending deformation due to buckling is one of the major collapse modes of the automotive structures [2]. Therefore, the performance of structural materials under bending deformation needs to be investigated carefully, especially at high rate of deformation.

TRIP (Transformation-induced Plasticity) steel is nowadays in widespread use in the automobile industry [3] because of favorable mechanical properties and high energy-absorption characteristic. Although, TRIP steel has particularly attracted the considerable attention of researchers [4-6], just few studies can be found on the bending deformation behavior and energy absorption of TRIP steel, especially under impact loading condition. Thus, an investigation of energy-absorption characteristic at high impact velocity for TRIP steel is indispensable expected from viewpoint of a safety design. On the

\footnotetext{
${ }^{a}$ Corresponding author: author@email .org
}

other hand, in general, energy-absorption characteristic of materials can be evaluated from a stress-strain curve obtained by a tensile test. However, an unstable deformation at high strain rate induces difficulties to determine onset of necking in a specimen, which is used to evaluate energy absorption. At the same time, threepoint bending test is considered to be relatively simple for investigation of performance of smooth specimen as well as becomes a useful tool in the determination of fracture parameters $[7,8]$. As a result, energy absorption in TRIP steel can be calculated from the three-point bending tests.

Previously, few studies have investigated experimentally on the energy absorption in TRIP steel. According to Rong et al. [9] and Curtze et al. [10], a rate sensitivity of energy absorption in TRIP steel can be observed from results of the tensile tests. Based on the observations of perforation tests, Rodríguez-Martínez et al. [11] reported that a decreasing testing temperature leads to larger values of the impact energy-absorption for TRIP steel. On the other hand, energy absorption characteristic during fracture process of materials is considered to be associated with fracture toughness $[12,13]$. Recently, Zhu and Joyce [14] evaluated energy absorption of materials by $J$ integral defined by Rice [15] from the area under a force-displacement as a result of the presence of a crack initiation.

In this study, the performance of both smooth and precracked specimens made of type 304 austenitic stainless steel, a kind of TRIP steel, is investigated experimentally at various deflection rate. The three-point bending tests are conducted by an INSTRON-type conventional material 
testing machines for quasi-static loading, a drop-weight testing machine, and a testing apparatus based on the split Hopkinson pressure bar (SHPB) method for smooth specimen. Experimental procedures for the three kinds of bending tests are described in detail. Then, the threepoint bending tests are conducted under quasi-static and impact conditions for pre-cracked specimen based on the ASTM standard. Energy absorption for TRIP steel during plastic deformation and fracture process will be discussed by examination of characteristic of force-deflection curves and value of $J$-integral. Additionally, stretch zone width is also investigated at different deflection rate to check the validity for the result of $J$-integral. Finally, conclusions on energy-absorption characteristic in TRIP steel are introduced.

\section{Methodology}

\subsection{Methodology to evaluate energy absorption characteristic}

In present study, energy absorption of smooth specimen is evaluated in terms of the increase in the external force because the area surrounding the normalized forcenormalized deflection curve increases until a certain level of normalized deflection is reached because of a ratesensitive hardening effect. Additionally, value of energyabsorption characteristic in fracture process is calculated by $J$-integral defined by Rice [10] from experimental results for pre-cracked specimen at the onset point of crack extension. Thus, it is necessary to determine an onset point of crack extension during deformation of the pre-cracked specimen. Here, a direct current potential drop technique is used for a detection of the onset of crack extension. A constant current is supplied through the specimen. The potential difference across the crack will change as the result of crack growth since a resistance increases because of a decrease in the cross-section of the specimen. Consequently, the output voltage may be related directly to area of the crack extension, and the onset point of crack growth is defined by the point that the tendency of a change in voltage.

Then, $J$-integral is evaluated by obeying ASTM E1820 [7] as following equation.

$$
J=\frac{K^{2}\left(1-v^{2}\right)}{E}+\frac{2 A_{p l}}{B\left(W-a_{0}\right)}
$$

where $E$ is Young's modulus, $v$ is Poisson's ratio and $A_{p l}$ is area under force-deflection curve as shown in Fig. 1. $K$ is calculated as,

$$
\begin{aligned}
K= & \frac{P S}{B W^{2}} \times 3\left(\frac{a_{o}}{W}\right)^{1 / 2} \times \\
& \frac{\left[1.99-\left(\frac{a_{0}}{W}\right)\left(1-\frac{a_{0}}{W}\right)\left(2.15-3.93\left(\frac{a_{0}}{W}\right)+2.7\left(\frac{a_{0}}{W}\right)^{2}\right)\right]}{2\left(1+2 \frac{a_{0}}{W}\right)\left(1-\frac{a_{0}}{W}\right)^{3 / 2}}
\end{aligned}
$$

where $P$ is external force, $S$ is span length, $B$ and $W$ are the initial thickness and width of the specimen, respectively, $a_{o}$ is the initial crack length including both the fatigue pre-cracked and the notch length.

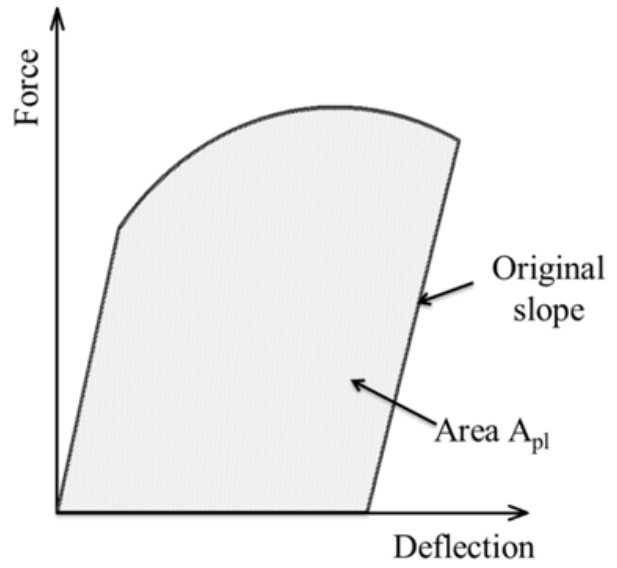

Figure 1. The definition of area under force-deflection curve for the calculation of $J$-integral [7].



Figure 2. The dimensions of the pre-cracked specimen.

\subsection{Specimens}

Smooth plate specimens (length: $100 \mathrm{~mm}$, height: $15 \mathrm{~mm}$, width: $7.5 \mathrm{~mm}$ ) and pre-cracked specimens with the dimensions obeyed the ASTM standard as shown in Fig. 2 are used in the present study. These specimens are made of type 304 austenitic stainless steel, a kind of TRIP steel. To obtain a uniform austenitic structure, after machining, the specimens are subjected to a solution-heat treatment at $1323 \mathrm{~K}$ for $30 \mathrm{~min}$ in an electric furnace (Full-tech FT100) and then quenched in cold water.

\subsection{Experimental apparatus}

\subsubsection{Quasi-static test}

Figure 3 shows a photograph of an Instron-type conventional material testing machine (Shimadzu AG$\mathrm{X} 250 \mathrm{KN}$ ) for quasi-static three-point bending test. The deflection rate is controlled by using constant crosshead speeds of $0.008,0.08,0.8$, and $8 \mathrm{~mm} / \mathrm{s}$ for smooth specimen and $36,360,3600,36000 \mathrm{~mm} / \mathrm{s}$ for pre-cracked specimen. Deflection is measured as the displacement of the crosshead of the testing machine. In addition, experimental apparatus to determine the onset of crack extension of pre-cracked specimen are shown in this figure. The location of probes for supplying current and measuring output voltage can be seen here. The probes for measuring output voltage are fixed around the cracktip on the opposite side in the thickness direction of the specimen to make the output voltage signals as sensitive as possible. They are spot-welded on the specimen. The supplying current in experiment is set to $12 \mathrm{~A}$ by a parallel 




Figure 3. A photograph of testing machine for quasi-static test.

connection of two DC power supplies (KENWOOD PA18$6 A)$.

\subsubsection{Drop-weight impact test}

Figure 4 shows a schematic illustration of the drop-weight testing setup.

A force-sensing block introduced by Chuman et al. [16] is placed under the center of the jig to capture the impact force for long time periods by protecting the reflected wave that propagates back into the force-sensing part. A low-pass filter (Nippon Avionics 9B02) with a cutoff frequency of $400 \mathrm{~Hz}$ is used to filter oscillations of output signals. A weight with a mass of $140 \mathrm{~kg}$ is allowed to freely fall from a height of $45 \mathrm{~mm}$ for smooth specimen and 400, 450 and $550 \mathrm{~mm}$ for pre-cracked specimen. The optical fiber sensors (Keyence FU-77V) and amplifiers (Keyence FS-V31 and 32) are used to measure the velocity of the weight just before it hits the specimen. Deflection is recorded using a high-speed camera (Photron Fastcam512PCI32K) during impact deformation with respect to time. A linear magnetic scale system including a magnetic scale (MACOME Laboratory SIS-310S) and a sensor head (MACOME Laboratory SIH-410) is used to measure the displacement of the weight. The deflection rate is calculated from the obtained deflection with respect to time by the finite difference method.

\subsubsection{Impact test based on split Hopkinson pressure bar method}

The impact test based on SHPB technique is used to investigate deformation behavior of smooth specimen at relatively high defection rate. The schematic diagram of the modified apparatus for the three-point bending test based on the SHPB method proposed by Yokoyama and Kishida [17] for smooth specimen is shown in Fig. 5. A striker bar (length: $500 \mathrm{~mm}$ ), an input bar (length: $4000 \mathrm{~mm}$ ), and two output bars (length: $4000 \mathrm{~mm}$ ) are used. All the pressure bars are $16 \mathrm{~mm}$ in diameter. The axial strain in the three pressure bars obtained from the gages is recorded using the above mentioned system. The velocity of the striker bar just before it hits the



Figure 4. A schematic illustration of the drop-weight test.

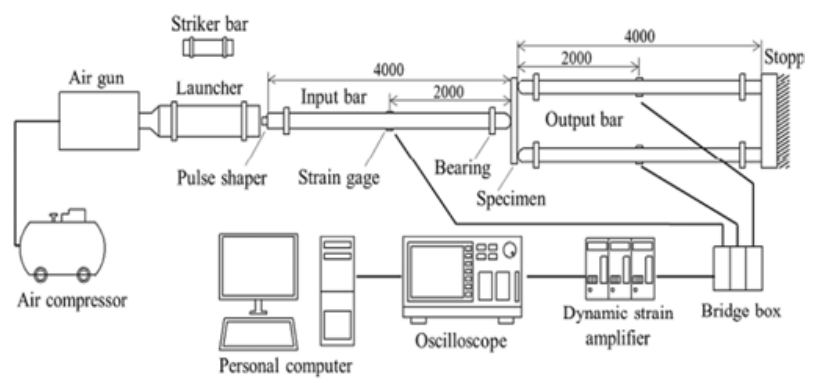

Figure 5. A schematic of the three-point bending test based on the SHPB method.

input bar is measured using the same system mentioned above. The impact velocity of the striker is controlled by controlling the pressure in the air tank. Air pressures of 2 and $5 \mathrm{MPa}$ correspond to impact velocities of 4000 and $8000 \mathrm{~m} / \mathrm{s}$, respectively. To suppress the initial oscillation of the transmitted and reflected stress waves, pulse shapers made of zinc are used. By using the incident, reflected, and transmitted stress pulses, the deflection at the center of the specimen is measured by a conventional method [17] and the deflection rate is calculated with respect to time by a finite difference method.

\subsection{Measurement of stretch zone width}

The plastic deformation at the crack tip leads to blunting the crack tip and finally the formation of the stretch zone. It is considered that the stretch zone width is strongly related to the resistance of the crack initiation [13]. Therefore, the stretch zone width is also investigated in present study to confirm validity of the value of $J$ integral. After bending deformation, the fracture surface of the pre-cracked specimen is observed by an analysis of fractography taken by a scanning electron microscope (SEM, JEOL, JSM-6510). If an image of fracture surface is at relatively-higher magnifications, then the crack stretch zone exhibits a typical featureless appearance easily distinguishable from the surface of the fatigue pre-crack. 


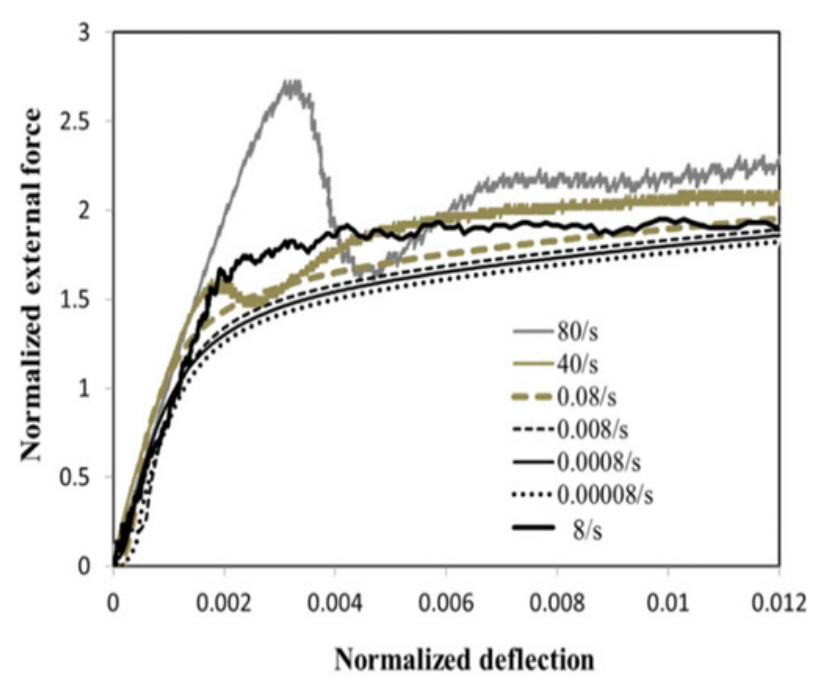

Figure 6. Normalized external force-normalized deflection curves obtained from the experiment for various normalized deflection rate for smooth specimen.

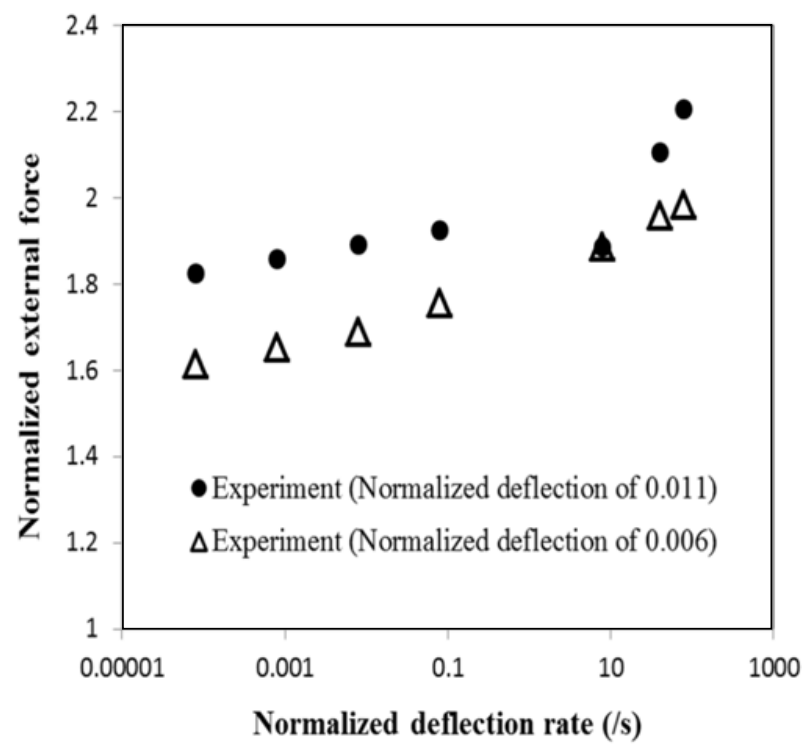

Figure 7. A plot of the normalized external force for a normalized deflection of 0.006 and 0.011 with respect to the normalized deflection rate.

Finally, the value of the stretch zone width is obtained by counting the pixels on the images.

\section{Results and discussion}

The measured external force and the deflection are normalized. An external force $P$ is normalized as the ratio of the maximum bending stress at the middle of the specimen to the initial yield stress at room temperature $\sigma_{y}$. The normalized force $P_{n o r}$, the normalized deflection $\delta_{n o r}$ and the normalized deflection rate $\dot{\delta}_{\text {nor }}$ are expressed as following equation.

$$
P_{n o r}=\frac{P L}{4 Z \sigma_{y}}, \delta_{n o r}=\frac{4 h \delta}{L^{2}}, \dot{\delta}_{n o r}=\frac{4 h \dot{\delta}}{L^{2}},
$$

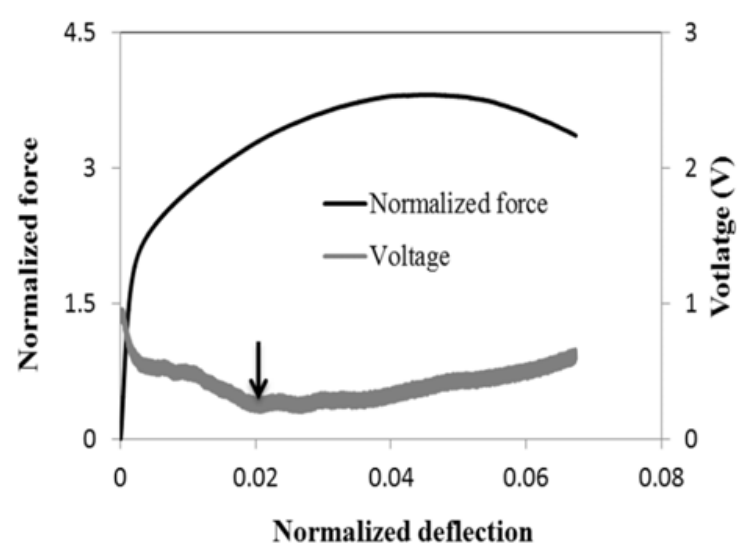

(a)

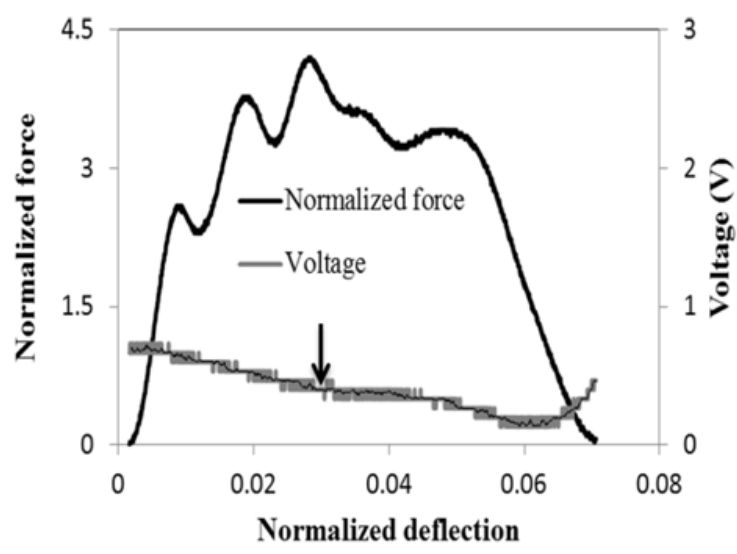

(b)

Figure 8. Force-deflection curve and determination of onset point of crack extension at normalized deflection rate of (a) $0.04 / \mathrm{s}$ and (b) $10.44 / \mathrm{s}$.

where $L$ is the span length and $Z$ is the section modulus, and $h$ is the height of the specimen. $\delta$ is deflection and $\dot{\delta}$ is the deflection rate obtained from experiment. In the case of smooth specimen, the corresponding normalized deflection rate is approximately $0.00008,0.0008,0.008$, and $0.08 / \mathrm{s}$ for the quasi-static deformation, and the maximum normalized deflection rate is approximately 8 , 40 , and $80 / \mathrm{s}$ for the impact deformation. These values of the normalized deflection rate are used as representative values for smooth specimen.

Figure 6 shows the normalized external forcenormalized deflection curves at various normalized deflection rate for smooth specimen. In cases of impact deformation, oscillations of external force are insufficiently suppressed although output signals are filtered by a low-pass filter in drop-weight impact test and the pulse shaper is used to generate incident wave in impact test based on SHPB method. Moreover, to obtain a certain level of normalized deflection, the normalized external force needs to be higher at higher normalized deflection rate. Next, value of the normalized external force for the normalized deflection of 0.006 and 0.011 at different normalized deflection rate is discussed.

Figure 7 shows the relationship between the normalized external force and normalized deflection rate in a semi-logarithmic plot for two cases of normalized 


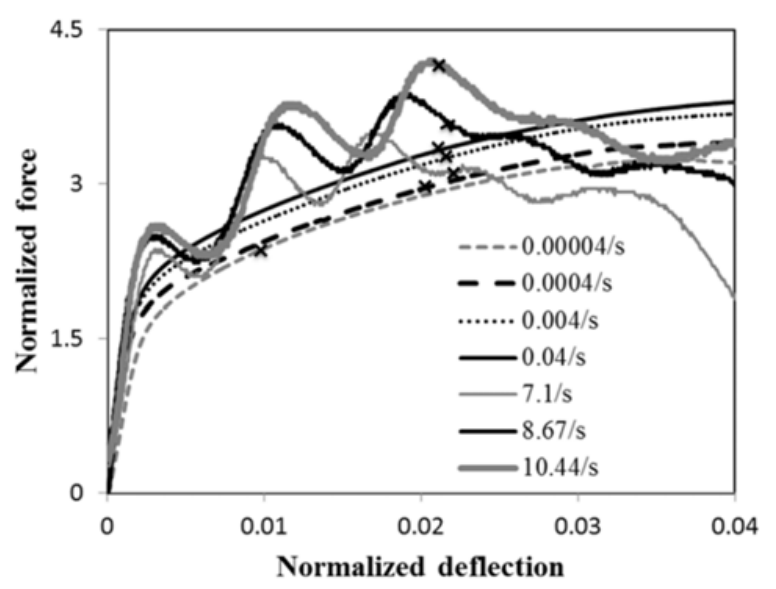

Figure 9. The relationship between normalized force and normalized deflection at various normalized deflection rate for pre-cracked specimen.

deflection. From this figure, TRIP steel might have higher energy absorption at a higher deflection rate. Moreover, a positive rate sensitivity of energy absorption in TRIP steel is clearly observed from quasi-static to impact deformation. An almost linear relationship between the normalized external force and normalized deflection rate can be seen at the lower level of normalized deflection. Thus, TRIP steel might show excellent characteristic of energy-absorption under impact loading condition. This can be explained that more energy might be consumed by inelastic deformation processes for a higher defection rate because of the hardening effect. The linear relationship between the normalized external force and normalized deflection rate in the cases of the quasi-static condition can be seen at higher normalized deflection. However, the slope of this relationship is slightly larger in the impact condition, and a sudden drop in the force can be seen for a normalized deflection rate of $8 / \mathrm{s}$. A higher temperature rise due to adiabatic heating by inelastic irreversible work at high deflection rate might induce thermal softening of materials in general. Furthermore, an increase in the temperature under impact condition at higher normalized deflection might suppress martensitic transformation in TRIP steel, which is expected to improve performance of this steel. Consequently, the relationship between normalized external force and normalized deflection rate becomes nonlinear. Therefore, it is predicted that the nonmonotonic positive rate sensitivity of the energy absorption in TRIP steel might not be observed with an increase in the normalized deflection.

In the case of pre-cracked specimen, the obtained normalized deflection rate is approximately 0.00004, 0.0004, 0.004 , and $0.04 / \mathrm{s}$ for the quasi-static deformation, and the maximum normalized deflection rate is approximately 7.1, 8.67 , and $10.44 / \mathrm{s}$ for the impact condition. They are also used as representative normalized deflection rate for precracked specimen.

Figure 8 shows the normalized force-normalized deflection curve and determination of the onset point of crack extension at normalized deflection rate of (a) $0.04 / \mathrm{s}$ and (b) 10.44/s. The change in the tendency of output voltage signals can be seen and is denoted by arrow in the

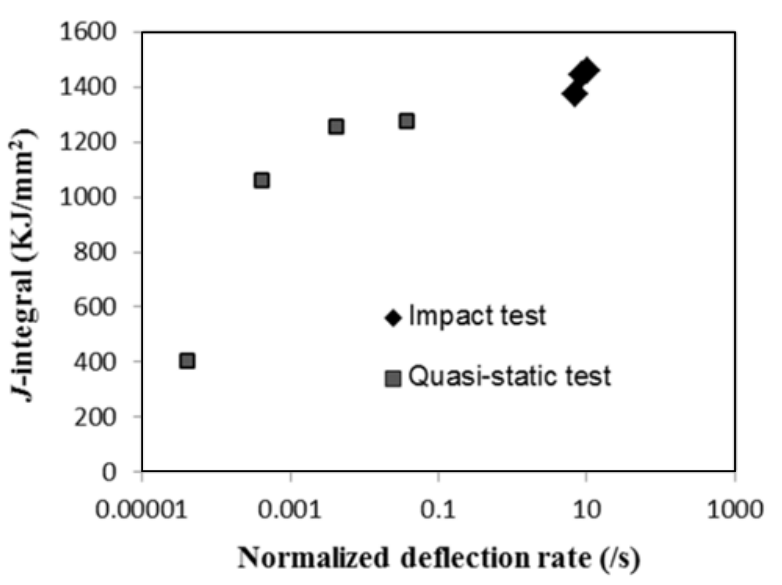

Figure 10. Value of $J$-integral at different normalized deflection rate.

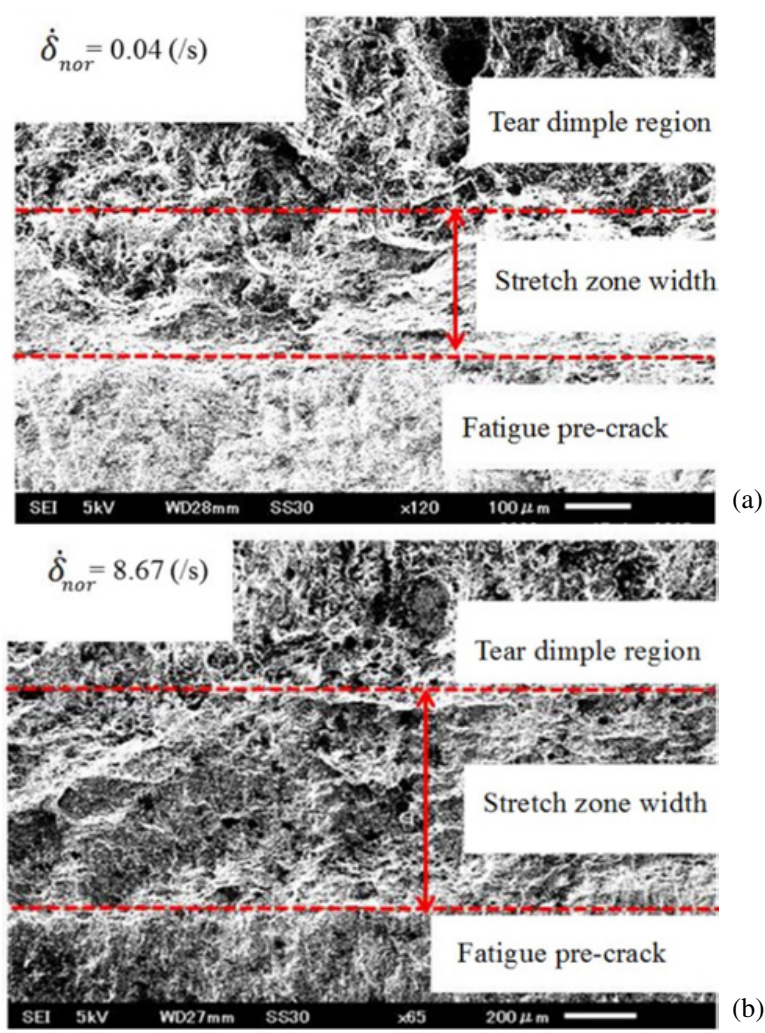

Figure 11. Photograph of microstructure near the crack tip including stretch zone in the case of normalized deflection rate of (a) $0.004 / \mathrm{s}$ and (b) $8.67 / \mathrm{s}$.

figure. The onset point of crack extension can be detected by the point of the change in the tendency.

Figure 9 shows the normalized force-normalized deflection curve for pre-cracked specimen at different normalized deflection rate. The onset point of crack extension for each case of normalized deflection rate is denoted by " $\mathbf{x}$ " on each curve. Oscillations of normalized force-normalized deflection curves in impact deformation are observed in this figure. Clearly, a similar tendency with values for smooth specimen can be seen here. To obtain the same value of normalized deflection, value of normalized force required higher at higher normalized deflection rate. 


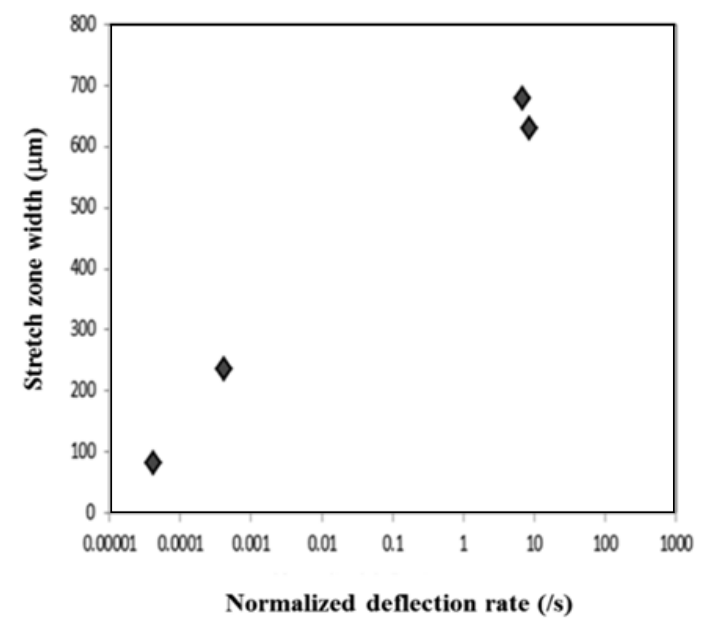

Figure 12. Relationship between stretch zone width and normalized deflection rate in a semi-logarithmic plot.

Additionally, value of the normalized deflection at the onset point of crack extension under impact loading is higher than that under quasi-static deformation in general. From this figure, energy absorption is calculated by $J$ integral. Then, the value of $J$-integral with respect to normalized deflection rate in a semi-logarithmic plot is shown in Fig. 10.

From Fig. 10, a higher value of $J$-integral can be seen at a higher normalized deflection rate with nonlinear relationship. Furthermore, this figure shows a positive rate sensitivity of $J$-integral from quasi-static to impact deformation. This result is suitable with observations from Fig. 7 for smooth specimen. As a result, TRIP steel might have better performance of energy absorption under impact loading condition.

Figure 11 shows photograph of the microstructure and definition of stretch zone width at normalized deflection rate of (a) 0.004/s under quasi-static deformation and (b) $8.67 / \mathrm{s}$ under impact loading deformation. From this figure, it can be seen clearly that the value of stretch zone width in impact case is relatively higher than that in quasi-static condition.

Figure 12 shows the value of stretch zone width at different normalized deflection rate. A considerably higher value of stretch zone width can be seen under impact condition than that under quasi-static deformation. Thus, a positive rate sensitivity of $J$-integral from quasi-static to impact deformation as above discussion can be confirmed.

\section{Concluding remarks}

In this study, the bending deformation behavior of both smooth and pre-cracked specimens made of TRIP steel is investigated experimentally at various deflection rate to evaluate energy-absorption characteristic. Characteristic of normalized force-normalized deflection curve and value of $J$-integral, which may be equivalent to the energy absorption, are discussed in quasi-static as well as impact condition. A positive rate sensitivity of the external force in TRIP steel can be observed from quasi-static to impact deformation. Moreover, TRIP steel indicates higher value of $J$-integral at higher normalized deflection rate. The positive rate sensitivity of $J$-integral is confirmed by an investigation of stretch zone width of fracture surface after bending deformation. As a result, it can be concluded that TRIP steel might have excellent energy-absorption characteristic under impact loading deformation.

We gratefully acknowledge financial supports from the $23^{\text {rd }}$ ISIJ Research Promotion Grant provided by the Iron and Steel Institute of Japan.

\section{References}

[1] Z. Kazanci, K. Bathe, Int. J. Imp. Eng. 42, 80 (2012)

[2] Z. Shen, X. Qiao, and H. Chen, SAE-china and FISITA (Eds), Proc. FISITA 2012 World Automotive Congress 197, 13 (2013)

[3] L. Durrenberger, D. Even, A. Molinari, A. Rusinek, J. Phys. IV. 134, 1287 (2006)

[4] T. Iwamoto, M. Cherkaoui, T. Sawa, Int. J. Modern Phys. B 22, 5985 (2008)

[5] F. D. Fischer, G. Reisner, E. Werner, K. Tanaka, G. Cailletaud, T. Antretter, Int. J. Plast. 16, 7231 (2000)

[6] Y. Tomita, T. Iwamoto, Int. J. Mech. Sci. 37, 1295 (1995)

[7] Standard Test Method for Measurement of Fracture Toughness, http://www.astm.org/ Standards/E1820

[8] J. F. Kalthoff, Int. J. Fract. 27, 277 (1985)

[9] T. Rong, L. Lin, B. C. De Cooman, W. Xi-chen, S. Peng, ISIJ Int. 13, 51 (2006)

[10] S. Curtze, V. T. Kuokkala, M. Hokka, P. Peura, Mater. Sci. Eng. A 507, 124 (2009)

[11] J.A. Rodríguez-Martínez, R. Pesci, A. Rusinek, A. Arias, R. Zaera, D. A. Pedroche, Int. J. Solids and Struct. 47, 1268 (2010)

[12] J. Nakayama, Japanese J. Appl. Phys. 3, 422 (1964)

[13] T. Iwamoto, T. Tsuta, Int. J. Plast. 18, 1583 (2002)

[14] X. K. Zhu, J. A. Joyce, U.S. Navy Res. Paper 49 (2012)

[15] J. R. Rice, J. Appl. Mech. 35, 379 (1968)

[16] Y. Chuman, K. Minuras, K. Kaizu, S. Tanimura, Int. J. Impact Eng. 19, 165 (1997)

[17] T. Yokoyama, K. Kishida, Exp. Mech. 29, 188 (1989) 